\title{
The Realization of the Parametric Excitation on MEMS Gyroscope
}

\author{
Lei Qian, Heming Zhao*, Dacheng Xu and Xiaofeng Zhang \\ School of Electronic and Information Engineering, Soochow University, Suzhou, China \\ ${ }^{*}$ Corresponding author
}

\begin{abstract}
In this paper, parametric excitation is applied to the excitation of a micro-ring gyroscope. The primary objective of this work is to improve the dynamic gain. It is show that, by adjusting the parametric excitation parameters, the harmonic forcing can be reduced whilst the gain of frequency response can be amplified. Because of the different frequencies of the excitation and harmonic forcing, the unwanted effect of electrical feed-through can keep in a minimum value.
\end{abstract}

Keywords-MEMS gyroscope; parametric excitation; closed-loop system

\section{INTRODUCTION}

MEMS (Micro-Electro-Mechanical System) gyroscopes have many advantages such as low cost, small size and negligible weight compared to the conventional mechanical gyroscopes. Furthermore, they have a wide range of applications including navigation and guidance systems, automobiles and consumer electronics, so that many researchers have focused on them for the last decades. However, to amplify the effective signal and reduce the noise remains the same.

Parametric resonance techniques have been used to amplify the mechanical signal in MEMS resonators, cantilevers and in MEMS gyroscopes. Parametric excitation refers to a method of excitation whereby a resonant system may be set into oscillation by periodically varying one of the parameters of the homogeneous system. Parametric amplification of approximately two orders of magnitude has been demonstrated in a forced mode of a micro-ring gyroscope [1]. When applied to the primary mode of the gyroscope, parametric amplification enables reduced forcing amplitudes and hence reduces electrical feed-through due to parasitic capacitances.

In this paper the experimental results from a MEMS gyroscope under high gain parametric amplification are described. The amplitude of the actuating signal may then be reduced so that the noise produced by electrical feed-through will be decreased. This will significantly improve the performance of the ring gyroscope.

\section{THEORY OF PARAMETRIC AMPLIFICATION}

The ring gyroscope mainly has two operating modes of drive and detection, and its dynamic model can be regarded as two order system of spring mass block with two degree of freedom. The system block diagram is shown in Figure 1.
As shown in figure $1, \mathrm{X}$ coordinate is drive modal movement direction, $\mathrm{Y}$ coordinate is sensitive modal movement direction. By applying voltage on the drive electrode, the mass block is driven by electrostatic force to do simple harmonic vibration in the $\mathrm{X}$ direction. When there is an angular rate input, due to the effect of the Coriolis force, mass produce relative displacement in the $\mathrm{Y}$ direction. The dynamic equation of two modes can be written [2, 3]:

$$
\left\{\begin{array}{c}
I_{x} \ddot{q}_{x}+\lambda_{x} \dot{q}_{x}+k_{x} q_{x}=F \\
I_{y} \ddot{q}_{y}+\lambda_{y} \dot{q}_{y}+k_{y} q_{y}=-2 I_{y} \Omega \dot{q}_{x}
\end{array}\right.
$$

In the formula, $I_{x}$ and $I_{y}$ are the rotational inertia of the drive mode and the sensitive mode respectively, $q_{x}$ and $q_{y}$ are displacement of mass blocks in driving modes and sensitive modes respectively, $\lambda_{x}$ and $\lambda_{y}$ are damping coefficients of drive modes and sensitive modes, respectively, $k_{x}$ and $k_{y}$ are the stiffness coefficients of the driving modes and the sensitive modes respectively.

The steady-state solutions of the drive mode are obtained by solving the upper modes:

$$
\begin{aligned}
& q_{x}=A \cos \left(\omega t-\varphi_{x}\right) \\
& A=\frac{M}{I_{x} \sqrt{\left(\omega_{x}^{2}-\omega^{2}\right)^{2}+\left(\omega \omega_{x} / Q_{x}\right)^{2}}} \\
& \varphi_{x}=\arctan \left(\frac{\omega_{x} \omega}{Q_{x}\left(\omega_{x}^{2}-\omega\right)}\right)
\end{aligned}
$$

Here, $\omega_{x}=\sqrt{k_{x} / I_{x}}$ is natural angular frequency of the drive mode. $Q_{x}=1 / 2 \delta_{x}$ is quality coefficient of drive mode. $\delta_{x}=\lambda_{x} /\left(2 I_{x} \omega_{x}\right)$ is damping ratio of drive mode. 


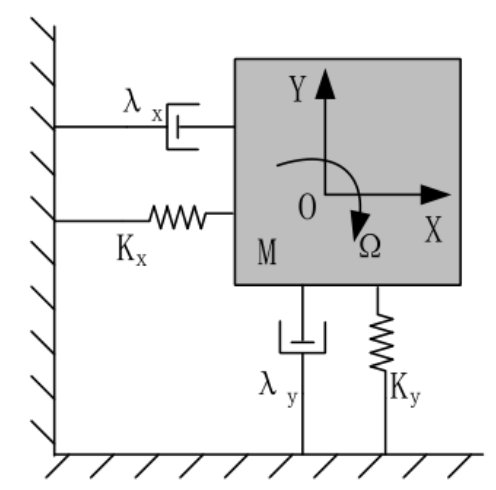

FIGURE I. MECHANICAL MODEL OF GYROSCOPE

A briefly description of parametric excitation and amplification for the MEMS gyroscope is presented in this section since a detailed description is available in $[4,5]$.

In order to enable parametric amplification of the MEMS gyroscope's response to harmonic forcing, the drive voltage $V_{d}(t)$ must be of the form:

$$
V_{d}(t)=V_{p} \sin \left(\omega_{p} t+\varphi\right)+V_{f} \sin \left(\omega_{f} t\right)
$$

Where: $\omega_{f}$ is the forcing frequency and $\omega_{p}=2 \omega_{f}, \varphi$ is the phase advance of parametric excitation with respect of the forcing in addition.

The linearized equation of the primary motion under combined harmonic forcing and parametric excitation has the form [6]:

$$
\ddot{q}+\frac{\omega_{\alpha}}{Q} \dot{q}+\left(\omega_{\alpha}{ }^{2}-\eta_{0}+\eta_{1} \sin \left(\omega_{p} t+\varphi\right)\right) q=-\bar{\eta}_{0} \sin \left(\omega_{f} t\right)
$$

With

$$
\begin{aligned}
& \eta_{0}=\frac{\varepsilon a d}{2 h_{0}^{3}}\left(\alpha+\frac{\sin (4 \alpha)}{4}\right)\left(V_{p}^{2}+V_{f}^{2}\right)+32 \alpha \frac{\varepsilon a d}{2 h_{0}^{3}} V_{d c}{ }^{2} \\
& \eta_{1}=4 \frac{\varepsilon a d}{2 h_{0}^{3}}\left(\alpha+\frac{\sin (4 \alpha)}{4}\right) V_{p} V_{d c} \\
& \bar{\eta}_{0}=2 \frac{\varepsilon a d}{2 h_{0}^{2}} \sin 2 \alpha V_{d c} V_{f}
\end{aligned}
$$

The solution of Eq. (4) can be written by

$$
q_{p}(t)=A \sin \left(\omega_{f} t+\psi\right)
$$

Where the steady state amplitude A and phase angle $\Psi$ of the primary mode with parametric excitation are describe

$$
\begin{aligned}
& A\left(V_{p}, \varpi, \varphi\right)=\bar{\eta}_{0} \frac{\sqrt{\varsigma^{2}+\eta_{1} \cos (\varphi)+\varpi^{4}+\eta_{1} \varpi^{2} \sin (\varphi)+\frac{1}{4} \eta_{1}^{2}}}{\varpi^{4}+\varsigma^{2}-\frac{1}{4} \eta_{1}^{2}} \\
& \psi\left(V_{p}, \varpi, \varphi\right)=\tan ^{-1}\left(-\frac{\varsigma+\frac{1}{2} \eta_{1} \cos (\varphi)}{\varpi^{2}+\frac{1}{2} \eta_{1} \sin (\varphi)}\right) \\
& \varpi^{2}=\varpi_{\alpha}^{2}-\omega_{f}^{2} \\
& \varpi_{\alpha}^{2}=\omega_{\alpha}^{2}-\eta_{0} \\
& \varsigma=\frac{\omega_{\alpha} \omega_{f}}{Q}
\end{aligned}
$$

Here, $\omega_{\alpha}$ represents the natural frequency of the ring gyroscope. $\bar{\omega}_{\alpha}$ represents the softened natural frequency because of the negative electrostatic stiffness caused by applied voltage so that the softened natural frequency is slightly less than the natural frequency.

The frequency response data could be represented in terms of the mobility $M\left(V_{p}, \varpi, \varphi\right)$, where [7]:

$$
\begin{aligned}
& \left|M\left(V_{P}, \varpi, \varphi\right)\right|=\frac{\omega_{f} A\left(V_{P}, \varpi, \varphi\right)}{\bar{\eta}_{0}} \\
& \theta=\psi\left(V_{P}, \varpi, \varphi\right)+\frac{\pi}{2}
\end{aligned}
$$

Gain, which can quantify the effect of parametric amplification, is defined as the ratio of the Mobility magnitude with parametric excitation 'ON' to the Mobility magnitude with parametric excitation 'OFF'.

$\operatorname{Gain}\left(V_{P}, \varpi, \varphi\right)=\frac{\left|A\left(V_{P}, \varpi, \varphi\right)\right|_{\text {on }}}{|A(\varpi)|_{\text {off }}}=\frac{\left|M\left(V_{P}, \varpi, \varphi\right)\right|_{\text {on }}}{|M(\varpi)|_{\text {off }}}$ (8)

\section{DEVICE DESCRIPTION}

The basic form of MEMS gyroscope is shown in figure 2 . Compared with the traditional MEMS ring gyro referenced in [8, $9,10]$, the gyro made some improvements. Two electrodes are placed between rings, rather than a single electrode, to increase the capacitance area. The positive and negative electrode formed the difference form, in order to achieve the goal of increasing the driving force and reducing noise. The dimensions are described in table 1.

The gyroscope is actuated and sensed electrostatically. Each of the electrode encompasses an angle $2 \alpha$. The gyroscope vibrates in an in-plane flexural mode. 
A dc voltage is applied to the ring structure. The driving signal is comprised by harmonic forcing and parametric excitation. The frequency of parametric excitation is twice as large as harmonic forcing, controlled by FPGA.

TABLE I. GYROSCOPE DIMENSIONS

\begin{tabular}{|c|c|}
\hline Parameter & Value \\
\hline Outer/Inner radius a1/a2 $(\mu \mathrm{m})$ & 1000 \\
\hline Thickness d $(\mu \mathrm{m})$ & 75 \\
\hline Width b $(\mu \mathrm{m})$ & 5.8 \\
\hline Capacitive gap ho $(\mu \mathrm{m})$ & 7.2 \\
\hline Q-factor & 25000 \\
\hline Natural frequency $(\mathrm{Hz})$ & 14870 \\
\hline Mass m(kg) & $1.46 \mathrm{e}-7$ \\
\hline $2 \alpha(\mathrm{rad})$ & $\pi / 4$ \\
\hline
\end{tabular}

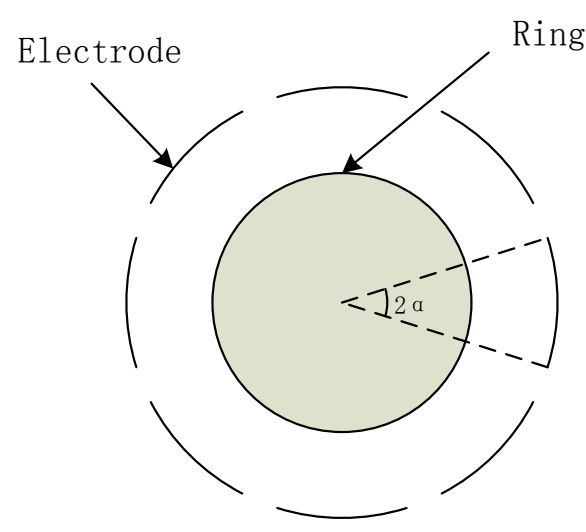

FIGURE II. BASIC FORM OF MEMS GUROSCOPE

\section{EXPERIMENTAL ARRANGEMENT}

Figure 3 shows the schematic layout of the experimental system used to assess the effects of parametric excitation on the MEMS gyroscope. This paper propose a MEMS resonant gyro closed-loop driving system based on FPGA. The closed-loop driving system of MEMS ring gyroscope consists of interface circuit, mixed analog/digital circuit and signal processing circuit as shown in figure 4 .

The function of the interface circuit is to translate the capacitance change caused by the mechanical vibration of the gyro drive mode into the test voltage, so as to realize the detection of the driving signal by the circuit. The mixed analog/digital circuit can be regarded as the connection device between the interface circuit and the FPGA digital system. The mechanical vibration of MEMS resonant gyroscope is converted into voltage and then sampled and quantized by ADC to become digital signal. The digital signal is processed by the algorithm designed in FPGA, and then converted into analog voltage through DAC to feedback on the gyro driving exciting electrode.

According to simulation analysis, the phase advance $\varphi$ of parametric excitation to harmonic forcing is a pronounced effect to the result of the experiment. In the simulation, it is obvious that the gain is most positive affected when the phase advance is zero. However, parametric excitation have side effect to gain when phase advance is close to $\pi$.Because of this, the phase advance $\varphi$ of parametric excitation to harmonic forcing in this experiment is controlled to become 0 .

Similarly, dc voltage applied to the gyroscope $V_{d c}$ is also related to the experimental result. In this experiment, $V_{d c}$ is determined to be $5 \mathrm{~V}$.

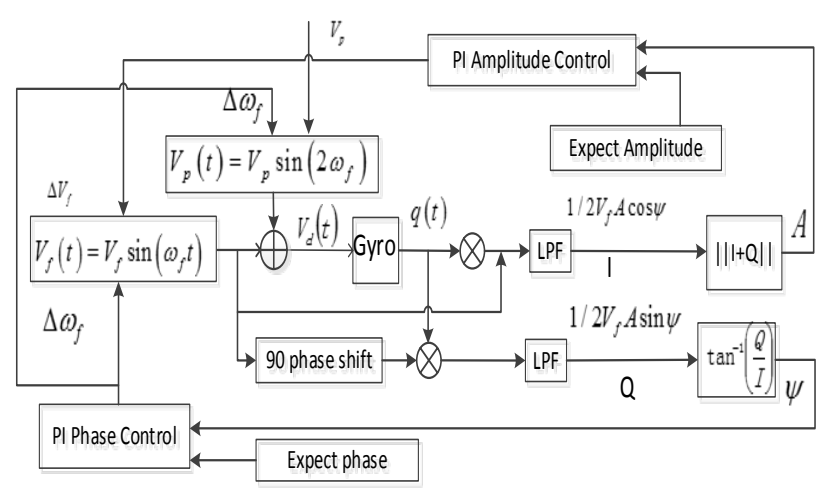

FIGURE III. CONTROL SCHEME

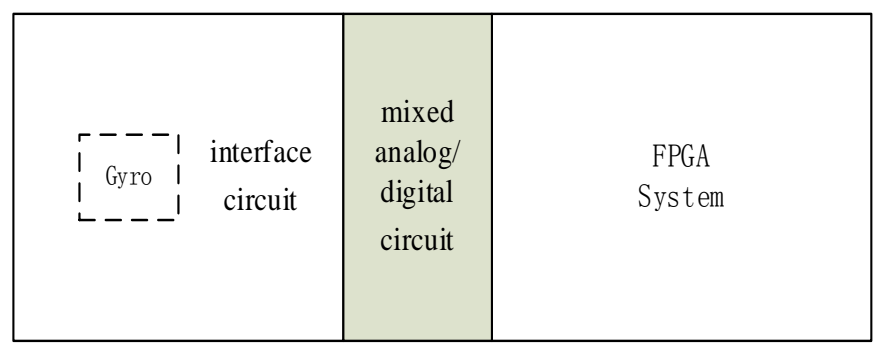

FIGURE IV. PCB MODEL OF THE TEST

\section{EXPERIMENTAL RESULTS FOR PARAMETRIC EXCITATION}

The closed loop experimental test results for the drive mode are shown in Figs. 5-6. Figure 5 shows the results of the test either with or without parametric excitation. As shown in this figure, the amplitude of the parametric is higher than drive signal to ensure the effect on the gain.

This is shown in Figure 5, the amplitude of harmonic forcing is decreased after adding the parametric excitation just like theory. 

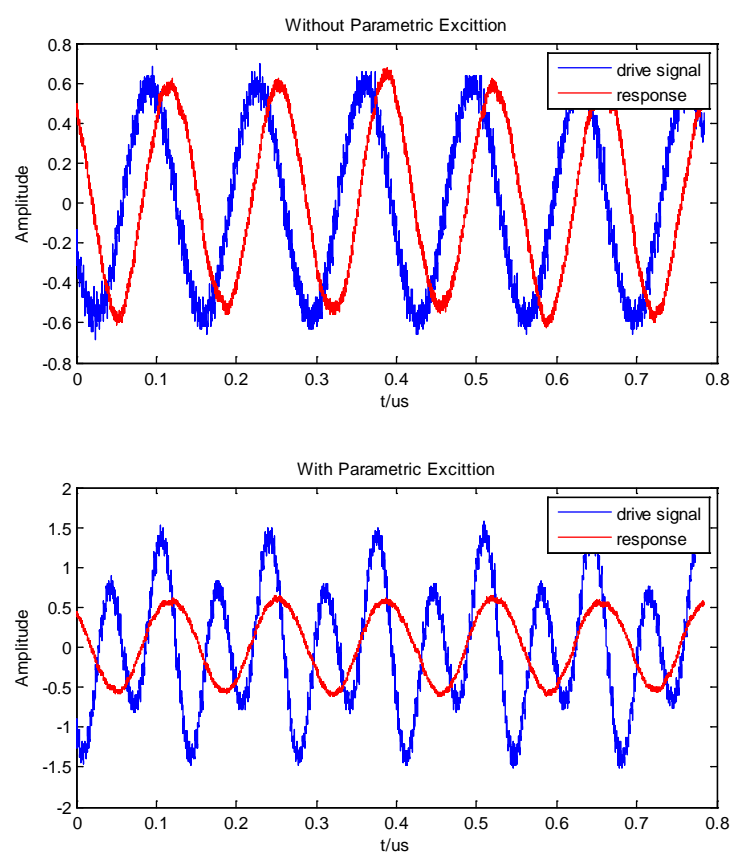

FIGURE V. THE RESULTS OF ADDING PARAMETRIC EXCITATION

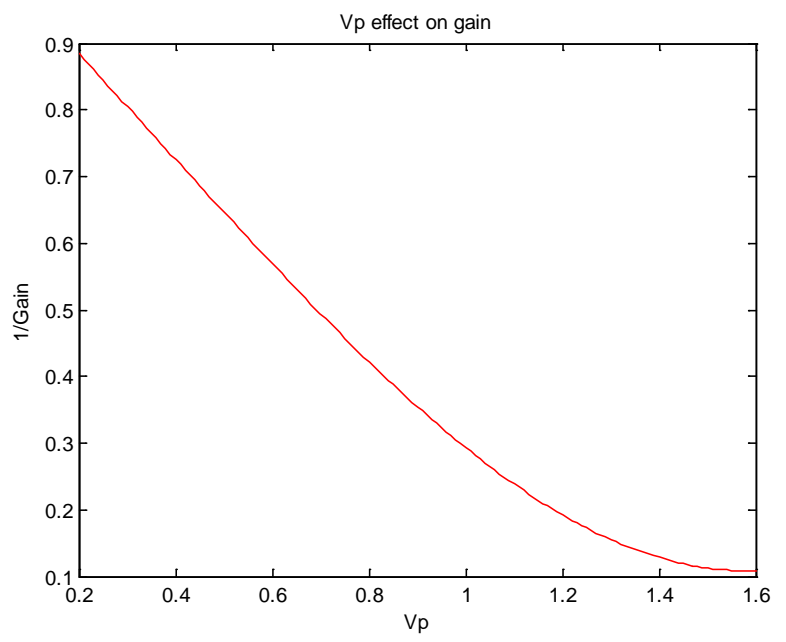

FIGURE VI. GAIN

Figure 6 shows the gain increase with $V_{p}$. From the figure, it can be found there is an obvious threshold about $V_{p}$ and gain. When $V_{p}<1.3 \mathrm{~V}$, there is a linear relationship between gain and $V_{p}$. However, when $V_{p}>1.3 \mathrm{~V}$, gain tends to a constant value. Hence, the amplitude of $V_{p}$ is limited to the linear range. In the linear range, with the rise of $V_{p}$, the ratio of the mobility magnitude with parametric excitation to the mobility magnitude without parametric excitation is rising correspondingly.

\section{CONCLUSION}

This paper research the influence of the parametric excitation signal on the gain. As shown in the above experimental results, parametric excitation is able to reduce the amplitude of harmonic forcing because it has positive effect on the gain.

It is clear that the amplitude of the substrate voltage and parametric excitation is related to the gain. It is desirable to improve the voltage limit by improving the equipment for further experiments. After that, the experimental results are expected to be further improved

Hence, the principal benefits of amplifying the drive mode is to enable reduced harmonic forcing level. Because of this, it is able to reduce the electrical feed-through of the drive signal to the sense electrodes. Then, it could improve the signal-tonoise ratio of the MEMS gyroscope.

\section{REFERENCES}

[1] B.J. Gallacher and J.S. Burdess, "Dynamic analysis of a microelectromechanical systems resonant gyroscope excited using combination parametric resonant,” Journal of Mechanical Engineering Science, vol.220, 2006, pp.1463-1479.

[2] Giorgio Casinovi, Arashk Norouzpour-Shirazi, Milap Dalal and Farrokh Ayazi, "Gyroscope sensing and self-calibration architecture based on signal phase shift," Sensors and Actuators A: Physical, vol.241,2016, pp.1-11.

[3] G.W. Hill, "On the part of the motion of lunar perigee which is a function of the mean motions of sun and moon," Act Mathematica, vol.8, 1886, pp.1-36.

[4] J.W. Strutt, "On the Maintenance of Vibrations by Forces of Double Frequency, and on the Propagation of Waves through a Medium endowed with a Periodic Structure,” Philosophical Magazine, vol.24, 1887, pp.145-159.

[5] J.S. Burdess, J. Hedly and B.J. Gallacher, "Electrostatic correction of structural imperfections present in a microring gyroscope,” Journal of Micromechanics and Microengineering, vol.14, 2005, pp.221-234.

[6] K.M. Harish and J.A. Neasham, "Simple parametric resonance in an electrostatically actuated microelectromechanical gyroscope: theory and experiment,” Journal of Mechanical Engineering Science, vol.222, 2008, pp.43-52.

[7] Zhongxu Hu, Barry J. Gallacher, Kiran M. Harish and James S. Burdess, "An experimental study of high gain parametric amplification in MEMS," Sensors and Actuators A: Physical, vol.162,2010, pp.145-154.

[8] K.M. Harish, and B.J. Gallacher, "A control scheme for a MEMS electrostatic resonant gyroscope excited using combined parametric excitation and harmonic forcing," Journal of Micromechanics and Microengineering, vol.16, 2006, pp.320-331.

[9] Z.X. Hu, C.P. Fell and K. Townsend, "A parametrically amplified MEMS rate gyroscope," Sensors and Actuators A: Physical, vol.167, 2011, pp.249-260.

[10] J.A. Neasham, B.J. Gallacher, J.S. Burdess and K.M. Harish, "Experimental investigation of parametric and externally forced motion in resonant MEMS sensors," Journal of Micromechanics and Microengineering, vol.19, 2009, pp.15-21. 